\title{
Evolved stars at high angular resolution: present and future
}

\author{
Claudia Paladini ${ }^{a}$ \\ ${ }^{a}$ Institut dAstronomie et dAstrophysique, Université libre de Bruxelles, CP 226, Boulevard du \\ Triomphe, 1050 Bruxelles, Belgium;
}

\begin{abstract}
The late evolutionary stages of stellar evolution are a key ingredient for our understanding in many fields of astrophysics, including stellar evolution and the enrichment of the interstellar medium (ISM) via stellar yields. Already the first interferometric campaigns identified evolved stars as the primary targets because of their extended and partially optically thin atmospheres, and the brightness in the infrared. Interferometric studies spanning different wavelength ranges, from visual to mid-infrared, have greatly increased our knowledge of the complex atmospheres of these objects where different dynamic processes are at play. In less than two decades this technique went from measuring simple diameters to produce the first images of stellar surfaces. By scanning the extended atmospheres we constrained theoretical models, learnt about molecular stratification, dust formation, and stellar winds, and there is still a lot to be done. In this contribution I will review the recent results that optical/infrared interferometry has made on our current understanding of cool evolved stars. The presentation will focus on asymptotic giant branch stars, and red supergiants. I will discuss the challenges of image reconstruction, and highlight how this field of research will benefit from the synergy of the current interferometric instrument(s) with the second generation VLTI facilities GRAVITY and MATISSE. Finally I will conclude with a short introspection on applications of a visible interferometer and of the the Planet Formation Imager (PFI) to the field of evolved stars.
\end{abstract}

Keywords: interferometry: scientific applications, future of interferometry, evolved stars, stellar atmospheres, imaging of stellar surfaces

\section{INTRODUCTION}

The stars covered in this review are the so-called asymptotic giant branch (AGB) and red supergiant stars (RSG). It is general knowledge that the initial mass on the main sequence will determine the fate of a star. Objects with a mass in the range 1-8 $M_{\odot}$, after going through the red giant and horizontal branch phase, will become AGB stars. During this phase, stars will expel their envelope via stellar wind, and end their lives as white dwarf. Stars more massive than 8-10 $M_{\odot}$ will have an almost horizontal excursion across the Hertzsprung-Russels diagram. After a quick phase as yellow supergiant they will become RSG, ending their lives with supernovae explosion. AGB and RSG stars have bolometric luminosity up to $10^{4} L_{\odot}$. The cool rarefied atmospheres have temperature in the range of $2000-4000 \mathrm{~K}$ and are the favoured place for the formation of various molecular and dust species. Both AGB and RSG stars are known to be a crucial player in the cosmic circuit of matter. Through the mass-loss process, these stars return the elements synthesised during their life to the interstellar medium. This material acts as building block for the next generation of stars, and mass loss is one of the main (still unknown) ingredients in stellar evolutionary codes.

Investigated since the early '70, the mass-loss process from evolved stars is still matter of discussion in the scientific community. Several open questions include: i) what drives the stellar wind in oxygen-rich AGB stellar atmospheres? ii) what drives the wind for the low-pulsating (or less evolved) AGBs? iii) what drives the wind in RSG? iv) what are the seeds for (oxygen-rich) dust formation; v) how does the stellar wind affects the various spatial scales of the stellar atmosphere (from the photosphere to the interface with the stellar envelope). This review will summarise where are we standing with the latter question.

I will focus on the "2-dimensional" information obtained over the last few years on the inner spatial scales probed with long baseline interferometry. With 2-dimensional information, I mean the possible asymmetric

For further information e-mail to paladini@ulb.ac.be 
structures that have been detected so far (via visibility model-fitting, direct closure phase and differential phase measurements), and their interpretation. Interested readers may want to consult also earlier reviews on the topic [123]. Results obtained with other interferometric techniques such as aperture masking or speckle interferometry are not covered here. I encourage the readers to look up very interesting recent results reported by Tuthill in the current proceeding (contribution 9907-13).

The review is divided in 4 main sections. The first part is dedicated to the studies conducted in the near-IR, where the photosphere and inner molecular envelope of giant stars (1-5 stellar radii) are observed. The second section will focus on the mid-IR studies, where dust formation occurs (up to $\sim 10$ stellar radii). The near-future prospects as well as more long-term forecasts will be given in the Section 4, followed by conclusing remarks in Section 5 .

\section{PHOTOSPHERE AND INNER MOLECULAR ENVIRONMENT}

Departure from circular symmetry has been known for giant stars from various high angular resolution observations [4, and references therein]. For many years the interpretation of such structures was not univocal and involved (i) spots due to convection, or (ii) elliptical distortion due to stellar rotation $[5$ and 6 are very recent examples]. However, at least in the case of AGB stars, high stellar rotation rates are usually connected to the presence of a binary companion transferring momentum to the primary [7, 8]. So far, convection appears to be the most likely explanation for the deviation from symmetry.

Despite what is observed on the Sun, where at least $10^{7}$ granules populate the surface, Schwarzschild [9] predicted the presence of only few convective cells on the stellar surface of AGB and RSG stars. In fact, a star evolving off the main sequence, inflates its radius and lower its gravity. Gravity together with temperature are the main parameters controlling the convection. When the star reaches the red giant branch the number of granules drops to a few thousand. The stellar surface becomes more and more irregular, and by the time the star is a RSG or an AGB, only a few convective patterns are expected to populate the stellar surface [10. As soon as the first images of RSG stars were available, the dichotomy between ellipticity vs. spots was solved. In [1] the stellar surface of Betelgeuse was imaged with IOTA. The images are reconstructed using WISARD 12 and MiRA [13] algorythms. A model prior and some regularization conditions are involved in the image reconstruction, but the two spots were confirmed via geometric model fitting of the visibilities. The authors mention that in analogy to what is observed in the Sun, the bright spots could be faculae, i.e. bright zones surrounded by dark convection cells. Connection with magnetic field is suggested, but could not be confirmed by these solo observations. Finally the same set of data was compared with 3D model predictions in [14 who confirmed the convective nature of the structures, and identified $\mathrm{H}_{2} \mathrm{O}$ as main opacity source in the image. Over the last years we have seen a few other cases of RSG images, and all of them had in common the detection of at least one large convective spot [15, 16, 17].

The first data sets allowing image reconstruction for AGB stars showed more or less asymmetric (molecular) shells [18, 19, 20, 21] with wavelength- and time-dependent global sizes. No obvious evidence of convective patterns are detected, however this is mainly the result of non optimal uv-coverage/visibility curve sampling. The recent image of $\mathrm{R}$ Car obtained from PIONIER data [17] confirms that spots are also detected on the surface of AGBs. At the time when this review is being written, at least three more stars have been imaged with PIONIER. Papers are in preparation [Paladini et al., Wittkowski et al.] and confirm the tendency of surface/convection related structures also in AGB stars. Preliminary results seems to support the idea that in the near-IR carbon-rich AGB stars are more asymmetric than their oxygen-rich counterpart [6].

Ascertained that convection is a crucial ingredient in the modelling of AGB and RSG, now one needs to determine: (i) what are the time-scales of convective patterns; (ii) how does the convection interplay with pulsation; (iii) what is the role of magnetic field. While 3D models are still under development [22, 23, 10], observational astronomers, and more precisely optical interferometrists, should push to obtain more images, and also to compare the observations with 1D available models. A summary of what has been done with 1D models is available in [3], nevertheless I would like to mention here a few interesting developments occurred in the field of RSG over the last few years. Usually 1D models successfully reproduce spectroscopy and photometric data. However this is not always the case for interferometric measurements, especially when it comes to RSG. 
Asymmetric structures can explain the disagreement only partially, as they mainly show up at high spatial frequencies. In a series of works [24, 25, 26] showed that PHOENIX hydrostatic models are too compact to reproduce the observed extension of RSG stars in the near-IR, while on the other hand they can reproduce the observations of low-pulsating AGBs. The use of 3D models including convection prescription does not improve the situation of RSG, and neither the 1D CODEX models implementing pulsation. A correlation between visibility ratio (continuum / CO (2-0) bandhead) of RSGs and the luminosity and surface gravity is observed. Extension of the atmospheres of RSG and mira stars are comparable 26. However the correlation between luminosity and surface gravity just mentioned is not observed in the case of mira variables. This points towards the possibility that the extension of the layers of RSG is not triggered by shocks generated during pulsation, as in the case of miras. The authors suggest that radiatively driven extension caused by radiation pressure on Doppler-shifted molecular lines might help to levitate the layers. As also magnetic field has been measured on the surface of RSG [27, it might very well be that in the end all these mechanisms have to be taken into account during modelling. Within this frame, simultaneous interferometric and polarimetric observations will help to clarify what is the interplay between magnetic field and convection [28, 29].

\section{THE DUST-FORMING REGION}

Both AGB and RSG show infrared dust excess in their spectra. The details of dust formation and acceleration process are matter of discussion [Sect. 3, 3]. Departure from spherical symmetry have been observed also in the dust forming region $(\sim 10 \mu \mathrm{m})$, at the onset of stellar wind. Asymmetric structures around AGB stars detected have been interpreted in terms of molecular or dust blobs 30, 31, 32, circumbinary/circum-companion discs 33. 34, 35, or as the signature of a binary companion [R Aqr, 36. The picture of RSG is similar to the one on the AGB: 37 interpreted asymmetric structures observed around Betelgeuse as related to the photospheric convective patterns, while 38 reported the presence of a pole-on torus around WOH G64. Although additional observations obtained with single-dish telescopes support the fact that both dust-plumes and torus are present around AGB and RSG [39, 40, 41, 42, it is obvious that there is no wide consensus on what really causes the departure from symmetry observed in the interferometric data. This is mainly due to the lack of imaging capabilities of the current (and past) interferometers in this wavelength range, but the scenario will improve as soon as VLTI/MATISSE will be on sky.

\section{OUTLOOK}

Since the early days of long baseline interferometry, AGB and RSG have been favourite targets for their high luminosity and extended atmospheres. Imaging is relatively challenging (see the contributions of Baron, Soulez, and Sanchez in this proceeding). The very complex environments and time variability require a good uv-coverage and sampling of the visibility curve, and the data cannot be combined from one year to the other. Observations should be collected and combined within one month for the most variable objects (i.e., mira stars). Current instrumentations with 4 - and 6-telescopes have shown that images can be done more or less routinely in the near-IR, but so far we were limited to few spectral channels or broad band.

GRAVITY, the second generation instrument recently installed at VLTI, will provide high spatial and spectral resolution. Spectra at each position of the stellar surface across the $K$-band will be available. $\mathrm{CO}, \mathrm{H}_{2} \mathrm{O}, \mathrm{OH}$, $\mathrm{HCN}, \mathrm{C}_{2}$, and $\mathrm{C}_{2} \mathrm{H}_{2}$ molecules will be mapped as well as the continuum. If with PIONIER and MIRC we can already image convective patterns, the spectral resolution will allow to localise and characterise these structures in more detail [43, 44, 45]. Time-series will allow to follow the evolution of such pattern and also the interplay with pulsation. The possibility of obtaining a simultaneous polarization information is quite intriguing, although very challenging according to the experts. Polarization measurements combined with the high spatial and spectral resolution would allow to clarify how much magnetic field plays a role in the convection theory for this class of stars.

MATISSE will open new spectral windows in the $L$ and $M$ bands, and will deliver images also in the $N$-band allowing to solve the dichotomy discs vs. blobs. Very extended targets such as the nearby AGBs and most of the RSG might well benefit from the aperture masking experiments available on NACO, SPHERE, and VISIR to complement and complete the visibility data sets of long baseline interferometry. Since a connection between 
apparent surface features and the morphology of the dust shell has not been established yet, and given the variability of the stars, we would benefit from simultaneous observations. In this frame an experiment able to combine the various wavelength ranges (PIONIER+GRAVITY+MATISSE) and collect them in one shot would be very welcome (so-called i-Shooter interferometer).

On a longer time-scale, an extension of the observing window to the visible will allow to probe the deeper layers of these giant stars, and to study the hot chromospheric plasma. However, to be successfull such a visible instrument will have to take into account that cool giant stars might be as faint as 7-13 mag in $V$-band. Long baselines of the order of $\mathrm{km}$ foreseen by PFI [Kraus et al., this proceeding] in the thermal infrared will allow a breakthrough in the field. Galactic globular clusters, and possibly the Magellanic Clouds might be easily reached. The interferometric community will have the chance in such case to give a strong contribution to questions such as how the mass-loss process depends on metallicity.

\section{CONCLUDING REMARKS}

In this contribution I briefly summarised the progress that long baselines interferometric studies have brought in the field of cool evolved stars, with a focus on the geometry of the environment. Time series of images at high spectral resolution for a benchmark of objects are needed to constrain the theory. Such kind of study needs to be coordinated with other multi-wavelengths and multi-technique observations in order to be able to tackle the main questions highlighted in the text. Given the complexity and the variable nature of these stars, time should be invested to investigate a polarization mode, and also to retrieve flux calibrated spectra/photometry from the current interferometric facilities.

\section{ACKNOWLEDGMENTS}

PC is supported by the Belgian Fund for Scientific Research F.R.S.- FNRS. I would like to acknowledge all my colleagues from the cool stars and interferometry community for many interesting discussions and crazy ideas.

\section{REFERENCES}

[1] Scholz, M., "Mira science with interferometry: a review," in [Interferometry for Optical Astronomy II], Traub, W. A., ed., Proc. SPIE 4838, 163-171 (Feb. 2003).

[2] Perrin, G., "Interferometry: The tool to study giant, supergiant and Mira stars," Ap\&SS 286, 197-212 (2003).

[3] Wittkowski, M. and Paladini, C., "From the atmosphere to the circumstellar environment in cool evolved stars," in [EAS Publications Series], EAS Publications Series 69, 179-195 (Sept. 2014).

[4] Ragland, S., Traub, W. A., Berger, J.-P., Danchi, W. C., Monnier, J. D., Willson, L. A., Carleton, N. P., Lacasse, M. G., Millan-Gabet, R., Pedretti, E., Schloerb, F. P., Cotton, W. D., Townes, C. H., Brewer, M., Haguenauer, P., Kern, P., Labeye, P., Malbet, F., Malin, D., Pearlman, M., Perraut, K., Souccar, K., and Wallace, G., "First Surface-resolved Results with the Infrared Optical Telescope Array Imaging Interferometer: Detection of Asymmetries in Asymptotic Giant Branch Stars," ApJ 652, 650-660 (Nov. 2006).

[5] van Belle, G. T., Paladini, C., Aringer, B., Hron, J., and Ciardi, D., "The PTI Carbon Star Angular Size Survey: Effective Temperatures and Non-sphericity," ApJ 775, 45 (Sept. 2013).

[6] Cruzalèbes, P., Jorissen, A., Chiavassa, A., Paladini, C., Rabbia, Y., and Spang, A., "Departure from centrosymmetry of red giants and supergiants measured with VLTI/AMBER," MNRAS 446, 3277-3284 (Feb. 2015).

[7] Barnbaum, C. and Hinkle, K. H., "Infrared and Optical Velocities of Carbon Stars," AJ 110, 805 (Aug. 1995).

[8] Mayer, A., Jorissen, A., Paladini, C., Kerschbaum, F., Pourbaix, D., Siopis, C., Ottensamer, R., Mečina, M., Cox, N. L. J., Groenewegen, M. A. T., Klotz, D., Sadowski, G., Spang, A., Cruzalèbes, P., and Waelkens, C., "Large-scale environments of binary AGB stars probed by Herschel. II. Two companions interacting with the wind of $\pi^{1}$ Gruis," A\&A 570, A113 (Oct. 2014). 
[9] Schwarzschild, M., "On the scale of photospheric convection in red giants and supergiants," ApJ 195, 137-144 (Jan. 1975).

[10] Chiavassa, A. and Freytag, B., "3D Hydrodynamical Simulations of Evolved Stars and Observations of Stellar Surfaces," in [Why Galaxies Care about AGB Stars III: A Closer Look in Space and Time], Kerschbaum, F., Wing, R. F., and Hron, J., eds., Astronomical Society of the Pacific Conference Series 497, 11 (Aug. 2015).

[11] Haubois, X., Perrin, G., Lacour, S., Verhoelst, T., Meimon, S., Mugnier, L., Thiébaut, E., Berger, J. P., Ridgway, S. T., Monnier, J. D., Millan-Gabet, R., and Traub, W., "Imaging the spotty surface of Betelgeuse in the H band," A\&A 508, 923-932 (Dec. 2009).

[12] Meimon, S., Mugnier, L. M., and Le Besnerais, G., "Convex approximation to the likelihood criterion for aperture synthesis imaging," Journal of the Optical Society of America A 22, 2348-2356 (Nov. 2005).

[13] Thiébaut, E., "MIRA: an effective imaging algorithm for optical interferometry," in [Optical and Infrared Interferometry], Proc. SPIE 7013, 70131I (July 2008).

[14] Chiavassa, A., Haubois, X., Young, J. S., Plez, B., Josselin, E., Perrin, G., and Freytag, B., "Radiative hydrodynamics simulations of red supergiant stars. II. Simulations of convection on Betelgeuse match interferometric observations," A\&A 515, A12 (June 2010).

[15] Chiavassa, A., Lacour, S., Millour, F., Driebe, T., Wittkowski, M., Plez, B., Thiébaut, E., Josselin, E., Freytag, B., Scholz, M., and Haubois, X., "VLTI/AMBER spectro-interferometric imaging of VX Sagittarii's inhomogenous outer atmosphere," A\&A 511, A51 (Feb. 2010).

[16] Baron, F., Monnier, J. D., Kiss, L. L., Neilson, H. R., Zhao, M., Anderson, M., Aarnio, A., Pedretti, E., Thureau, N., ten Brummelaar, T. A., Ridgway, S. T., McAlister, H. A., Sturmann, J., Sturmann, L., and Turner, N., "CHARA/MIRC Observations of Two M Supergiants in Perseus OB1: Temperature, Bayesian Modeling, and Compressed Sensing Imaging," ApJ 785, 46 (Apr. 2014).

[17] Monnier, J. D., Berger, J.-P., Le Bouquin, J.-B., Tuthill, P. G., Wittkowski, M., Grellmann, R., Müller, A., Renganswany, S., Hummel, C., Hofmann, K.-H., Schertl, D., Weigelt, G., Young, J., Buscher, D., Sanchez-Bermudez, J., Alberdi, A., Schoedel, R., Köhler, R., Soulez, F., Thiébaut, É., Kluska, J., Malbet, F., Duvert, G., Kraus, S., Kloppenborg, B. K., Baron, F., de Wit, W.-J., Rivinius, T., and Merand, A., "The 2014 interferometric imaging beauty contest," in [Optical and Infrared Interferometry IV], Proc. SPIE 9146, 91461Q (July 2014).

[18] Ragland, S., Le Coroller, H., Pluzhnik, E., Cotton, W. D., Danchi, W. C., Monnier, J. D., Traub, W. A., Willson, L. A., Berger, J.-P., and Lacasse, M. G., "First Images of R Aquarii and Its Asymmetric $\mathrm{H}_{2} \mathrm{O}$ Shell," ApJ 679, 746-761 (May 2008).

[19] Lacour, S., Thiébaut, E., Perrin, G., Meimon, S., Haubois, X., Pedretti, E., Ridgway, S. T., Monnier, J. D., Berger, J. P., Schuller, P. A., Woodruff, H., Poncelet, A., Le Coroller, H., Millan-Gabet, R., Lacasse, M., and Traub, W., "The Pulsation of $\chi$ Cygni Imaged by Optical Interferometry: A Novel Technique to Derive Distance and Mass of Mira Stars," ApJ 707, 632-643 (Dec. 2009).

[20] Le Bouquin, J.-B., Lacour, S., Renard, S., Thiébaut, E., Merand, A., and Verhoelst, T., "Pre-maximum spectro-imaging of the Mira star T Leporis with AMBER/VLTI," A\&A 496, L1-L4 (Mar. 2009).

[21] Haubois, X., Wittkowski, M., Perrin, G., Kervella, P., Mérand, A., Thiébaut, E., Ridgway, S. T., Ireland, M., and Scholz, M., "Resolving asymmetries along the pulsation cycle of the Mira star X Hydrae," A\&A 582, A71 (Oct. 2015).

[22] Freytag, B. and Höfner, S., "Three-dimensional simulations of the atmosphere of an AGB star," A\&A 483, 571-583 (May 2008).

[23] Freytag, B., "Studying the Generation of Shock Waves in AGB Stars with 3-Dimensional RadiationHydrodynamics Simulations," in [Why Galaxies Care about AGB Stars III: A Closer Look in Space and Time], Kerschbaum, F., Wing, R. F., and Hron, J., eds., Astronomical Society of the Pacific Conference Series 497, 23 (Aug. 2015).

[24] Arroyo-Torres, B., Wittkowski, M., Marcaide, J. M., and Hauschildt, P. H., "The atmospheric structure and fundamental parameters of the red supergiants AH Scorpii, UY Scuti, and KW Sagittarii," A\&A 554, A76 (June 2013). 
[25] Arroyo-Torres, B., Martí-Vidal, I., Marcaide, J. M., Wittkowski, M., Guirado, J. C., Hauschildt, P. H., Quirrenbach, A., and Fabregat, J., "VLTI/AMBER observations of cold giant stars: atmospheric structures and fundamental parameters," A\&A 566, A88 (June 2014).

[26] Arroyo-Torres, B., Wittkowski, M., Chiavassa, A., Scholz, M., Freytag, B., Marcaide, J. M., Hauschildt, P. H., Wood, P. R., and Abellan, F. J., "What causes the large extensions of red supergiant atmospheres?. Comparisons of interferometric observations with 1D hydrostatic, 3D convection, and 1D pulsating model atmospheres," A\&A 575, A50 (Mar. 2015).

[27] Aurière, M., Donati, J.-F., Konstantinova-Antova, R., Perrin, G., Petit, P., and Roudier, T., "The magnetic field of Betelgeuse: a local dynamo from giant convection cells?," A\&A 516, L2 (June 2010).

[28] Montargès, M., Kervella, P., Perrin, G., Chiavassa, A., Le Bouquin, J.-B., Aurière, M., López Ariste, A., Mathias, P., Ridgway, S. T., Lacour, S., Haubois, X., and Berger, J.-P., "The close circumstellar environment of Betelgeuse. IV. VLTI/PIONIER interferometric monitoring of the photosphere," A\&A 588, A130 (Apr. 2016).

[29] Aurière, M., López Ariste, A., Mathias, P., Lèbre, A., Josselin, E., Montargès, M., Petit, P., Chiavassa, A., Paletou, F., Fabas, N., Konstantinova-Antova, R., Donati, J.-F., Grunhut, J. H., Wade, G. A., Herpin, F., Kervella, P., Perrin, G., and Tessore, B., "Discovery of a complex linearly polarized spectrum of Betelgeuse dominated by depolarization of the continuum," A\&A 591, A119 (June 2016).

[30] Chandler, A. A., Tatebe, K., Hale, D. D. S., and Townes, C. H., "The Radiative Pattern and Asymmetry of IRC +10216 at $11 \mu \mathrm{m}$ Measured with Interferometry and Closure Phase," ApJ 657, 1042-1045 (Mar. 2007).

[31] Paladini, C., Sacuto, S., Klotz, D., Ohnaka, K., Wittkowski, M., Nowotny, W., Jorissen, A., and Hron, J., "Detection of an asymmetry in the envelope of the carbon Mira R Fornacis using VLTI/MIDI," A\&A 544, L5 (Aug. 2012).

[32] Sacuto, S., Ramstedt, S., Höfner, S., Olofsson, H., Bladh, S., Eriksson, K., Aringer, B., Klotz, D., and Maercker, M., "The wind of the M-type AGB star RT Virginis probed by VLTI/MIDI," A\&A 551, A72 (Mar. 2013).

[33] Deroo, P., van Winckel, H., Verhoelst, T., Min, M., Reyniers, M., and Waters, L. B. F. M., "The circumbinary disc around the J-type C-star IRAS 18006-3213," A\&A 467, 1093-1101 (June 2007).

[34] Ohnaka, K., Izumiura, H., Leinert, C., Driebe, T., Weigelt, G., and Wittkowski, M., "Asymmetric silicate dust distribution toward the silicate carbon star BM Geminorum," A\&A 490, 173-178 (Oct. 2008).

[35] Klotz, D., Sacuto, S., Kerschbaum, F., Paladini, C., Olofsson, H., and Hron, J., "The geometry of the close environment of SV Piscium as probed by VLTI/MIDI," A\&A 541, A164 (May 2012).

[36] Tatebe, K., Chandler, A. A., Hale, D. D. S., and Townes, C. H., "Characterization of Dust Shell Dynamics and Asymmetry for Six Mira-Type Stars," ApJ 652, 666-680 (Nov. 2006).

[37] Ravi, V., Wishnow, E. H., Townes, C. H., Lockwood, S., Mistry, H., and Tatebe, K., "The Non-uniform, Dynamic Atmosphere of Betelgeuse Observed at Mid-infrared Wavelengths," ApJ 740, 24 (Oct. 2011).

[38] Ohnaka, K., Driebe, T., Hofmann, K.-H., Weigelt, G., and Wittkowski, M., "Spatially resolved dusty torus toward the red supergiant WOH G64 in the Large Magellanic Cloud," A\&A 484, 371-379 (June 2008).

[39] de Wit, W. J., Oudmaijer, R. D., Fujiyoshi, T., Hoare, M. G., Honda, M., Kataza, H., Miyata, T., Okamoto, Y. K., Onaka, T., Sako, S., and Yamashita, T., "A Red Supergiant Nebula at $25 \mu \mathrm{m}$ : Arcsecond-Scale MassLoss Asymmetries of $\mu$ Cephei," ApJ Letters 685, L75 (Sept. 2008).

[40] Kervella, P., Verhoelst, T., Ridgway, S. T., Perrin, G., Lacour, S., Cami, J., and Haubois, X., "The close circumstellar environment of Betelgeuse. Adaptive optics spectro-imaging in the near-IR with VLT/NACO," A\&A 504, 115-125 (Sept. 2009).

[41] Kervella, P., Perrin, G., Chiavassa, A., Ridgway, S. T., Cami, J., Haubois, X., and Verhoelst, T., "The close circumstellar environment of Betelgeuse. II. Diffraction-limited spectro-imaging from 7.76 to $19.50 \mu \mathrm{m}$ with VLT/VISIR," A\&A 531, A117 (July 2011).

[42] Ohnaka, K., "Imaging the outward motions of clumpy dust clouds around the red supergiant Antares with VLT/VISIR," A\&A 568, A17 (Aug. 2014). 
[43] Ohnaka, K., Weigelt, G., Millour, F., Hofmann, K.-H., Driebe, T., Schertl, D., Chelli, A., Massi, F., Petrov, R., and Stee, P., "Imaging the dynamical atmosphere of the red supergiant Betelgeuse in the $\mathrm{CO}$ first overtone lines with VLTI/AMBER," A\&A 529, A163 (May 2011).

[44] Ohnaka, K., Hofmann, K.-H., Schertl, D., Weigelt, G., Baffa, C., Chelli, A., Petrov, R., and Robbe-Dubois, S., "High spectral resolution imaging of the dynamical atmosphere of the red supergiant Antares in the CO first overtone lines with VLTI/AMBER," A\&A 555, A24 (July 2013).

[45] Ohnaka, K., Weigelt, G., and Hofmann, K.-H., "Clumpy dust clouds and extended atmosphere of the AGB star W Hydrae revealed with VLT/SPHERE-ZIMPOL and VLTI/AMBER,” A\&A 589, A91 (May 2016). 